05,01

\title{
ЯМР-исследование магнитной структуры и сверхтонких взаимодействий в бинарном гелимагнетике FeP
}

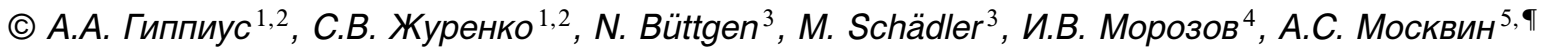 \\ ${ }^{1}$ Московский государственный университет им. М.В. Ломоносова, \\ Москва, Россия \\ ${ }^{2}$ Физический институт РАН им. П.Н. Лебедева, \\ Москва, Россия \\ ${ }^{3}$ Experimental Physics V, University of Augsburg, \\ Augsburg, Germany \\ ${ }^{4}$ Московский государственный университет им. М.В. Ломоносова, \\ Москва, Россия \\ ${ }^{5}$ Уральский фредеральный университет, \\ Екатеринбург, Россия \\ ๑ E-mail: alexander.moskvin@urfu.ru
}

\begin{abstract}
Проведено исследование ЯМР ядер ${ }^{31} \mathrm{P}$ в металлическом фосфиде железа FeP как в нулевом поле, так и путем развертки внешнего магнитного поля на нескольких фиксированных частотах с главной целью - выявить и исследовать трансформацию магнитной спиральной структуры FеР. Форма ЯМРлиний постепенно изменяется с увеличением поля от трапециевидной формы в низких полях до ярко выраженной асимметричной формы с двумя „рогами“ в сильных полях. Первая характерна для спектров ЯМР в порошкообразных образцах в приложенном магнитном поле, а последняя - для ЯМР-спектров на немагнитных атомах в монокристаллических гелимагнетиках. Наблюдаемая трансформация ЯМР-спектра FeP является убедительным свидетельством в пользу спин-переориентационного перехода типа спин-флоп в $\mathrm{FeP}$ в области больших внешних полей.
\end{abstract}

Работа выполнена при поддержке Программы 211 Правительства Российской Федерации, соглашение № 02.А03.21.0006, проекта РФФИ № 17-52-80036 и проектов № 2277 и № 5719 Министерства Образования и Науки Российской Федерации, а также Межрегиональной программы Немецкого Физического Общества TRR80 (Мюнхен, Аугсбург).

DOI: $10.21883 /$ FTT.2019.05.47576.23F

\section{1. Введение}

Научный интерес к фосфиду $\mathrm{FeP}$ во многом связан с внутренней сложностью его необычной спиральной магнитной структуры, происхождение и детали которой все еще являются предметом дискуссий. Кроме того, FeP заслуживает более пристального внимания из-за сходства структуры взаимодействий со сверхпроводниками на основе FeAs.

Фосфид FeP имеет орторомбическую структуру с пространственной группой Рпта при комнатной температуре [1]. Структура состоит из ионов железа, которые занимают эквивалентные кристаллографические позиции с окружающими атомами фосфора, образующими искаженные октаэдры $\mathrm{FeP}_{6}$, с четырьмя формульными единицами на элементарную ячейку. Согласно магнитным данным [2], это соединение обнаруживает магнитный переход при $T_{\mathrm{N}} \approx 120 \mathrm{~K}$ к двойной антиферромагнитной спирали с магнитными моментами, лежащими в $a b$-плоскости. В соответствии с геликоидальной структурой, предложенной в работе [3], магнитные моменты железа параллельны плоскостям, нормальным к $c$-оси.

Согласно результатам, полученным в [3], магнитная структура FeP может быть описана с учетом двух неэквивалентных позиций железа с различными магнит-

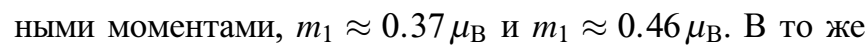
время в случае изоструктурного арсенида $\mathrm{FeAs}$ с почти одинаковой магнитной структурой [4] поляризованное рассеяние нейтронов показало, что магнитный геликоид имеет эллиптическую деформацию в $a b$-плоскости с самой длинной осью эллипса, направленной вдоль $b$-оси. Следует отметить, что порошковая нейтронная дифракция, применяемая для изучения магнитных свойств железа в пниктидах FeP и FeAs, не может отличить простую двойную спиральную модель с двумя дискретными позициями железа от квазинепрерывного эллиптически поляризованного распределения моментов железа, что предполагает использование альтернативных методик для однозначного решения вопроса о структуре спирали.

Ранние исследования эффекта Мессбауэра для ядер ${ }^{57} \mathrm{Fe}$, выполненные для $\mathrm{FeP}[4,5]$ в парамагнитной области температур $\left(T>T_{\mathrm{N}}\right)$, показали, что все катионы железа занимают одинаковые кристаллографические позиции. Однако идентификация и самосогласованный анализ очень сложных магнитных сверхтонких спектров при $T<T_{\mathrm{N}}$ вызвали серьезные трудности [5] и оставили ряд вопросов. Одно из наиболее полных мессбауэровских исследований ${ }^{57} \mathrm{Fe}$ монокристаллов FеР во внешнем магнитном поле было выполнено в работе [4]. Разумная 
подгонка была получена с помощью суперпозиции из нескольких зеемановских вкладов с различными значениями магнитных сверхтонких полей на ядрах ${ }^{57} \mathrm{Fe} . \mathrm{Co}-$ гласно [4], каждый дискретный зеемановский спектр соответствует различным ориентациям магнитного сверхтонкого поля в $a b$-плоскости. Было показано, что для хорошей подгонки необходимо учитывать „пучкование“ (bunching) спинов вдоль определенных направлений в $a b$-плоскости. К сожалению, авторы не обсуждали происхождение этого эффекта в контексте электронной структуры ионов железа в FеР. Более того, нет информации о температурной эволюции сверхтонкой зеемановской структуры месс бауэровских спектров, в частности вблизи критической точки $\left(T \approx T_{\mathrm{N}}\right)$, что было бы очень полезно для выяснения природы магнитного фазового перехода.

В работе Соболева и др. [6] представлены результаты исследования эффекта Мессбауэра для ядер ${ }^{57} \mathrm{Fe}$ в порошкообразных образцах фосфида железа FeP, выполненного в широком температурном диапазоне, включая температуру $\left(T_{\mathrm{N}} \approx 120 \mathrm{~K}\right)$ магнитного фазового перехода. Мессбауэровские спектры ${ }^{57} \mathrm{Fe}$ при низких температурах $T<T_{\mathrm{N}}$ представляют собой очень сложную зеемановскую картину с уширенными линиями и значительной спектральной асимметрией. Показано, что изменение наблюдаемой спектральной формы согласуется с переходом в пространственно-модулированную геликоидальную магнитную структуру. Анализ экспериментальных спектров проводился в предположении анизотропии магнитного сверхтонкого поля $H_{h f}$ на ядрах ${ }^{57} \mathrm{Fe}$, когда магнитный момент ионов железа вращается относительно главной оси тензора градиента электрического поля (ГЭП). Результаты показали, что хорошая подгонка экспериментальных спектров может быть достигнута без предположений о формировании двух неэквивалентных кристаллографических положений катионов железа, как это было предложено ранее. Основной причиной наблюдаемой асимметрии сверхтонкой структуры является пространственная модуляция углов поворота $\omega$ между соседними моментами $m_{\mathrm{Fe}}$ вдоль $c$-оси, что индуцирует модуляцию собственных значений гамильтониана сверхтонких взаимодействий.

Полученный аномально большой температурно независимый параметр ангармонизма $m \approx 0.96$ для геликоидальной спиновой структуры обусловлен сильной анизотропией типа „легкая ось““ в плоскости вращения спинов железа. Авторы предположили, что очень малую максимальную величину $H_{h f}(11 \mathrm{~K}) \approx 36 \mathrm{kOe}$ и ее высокую анизотропию $H_{\text {ani }}(11 \mathrm{~K}) \approx 30 \mathrm{kOe}$ можно объяснить стабилизацией катионов железа в низкоспиновом состоянии.

В целом вопрос о структуре геликоида в $\mathrm{FeP}$ и его эволюции в магнитном поле остается дискуссионным. С целью выявить и исследовать трансформацию магнитной спиральной структуры $\mathrm{FeP}$ в настоящей работе мы провели исследование ЯМР ядер ${ }^{31} \mathrm{P}$ в металлическом фосфиде железа FeP как в нулевом поле, так и путем развертки внешнего магнитного поля на нескольких фиксированных частотах.

\section{2. Экспериментальные результаты}

Образец поликристаллического FeP был синтезирован с использованием стандартной методики в вакуумированной кварцевой ампуле. Стехиометрическую смесь порошков $\mathrm{Fe}$ и $\mathrm{P}$ (красного) медленно нагревали до $1123 \mathrm{~K}$ в течение $30 \mathrm{~h}$ и отжигали при этой температуре в течение $48 \mathrm{~h}$, после чего печь выключали. Порошковый рентгенографический анализ проводили с использованием дифрактометра Bruker D8 Advance (излучение $\mathrm{Cu}-K_{\alpha 1}$, монохроматор Ge-111, геометрия отражения), оснащенного кремниевым полосковым LynxEye детектором. XRD-спектры подтвердили, что полученный образец представляет собой однофазный порошок FeP c орторомбической элементарной ячейкой: $a=5.203(1) \AA$, $b=3.108(1) \AA$ и $c=5.802(1) \AA$, пространственная группа Pnma, в согласии с литературными данными $[3,4]$. Чтобы предотвратить поверхностное окисление во влажном воздухе, все операции проводились в сухом боксе $\left.\left(p \mathrm{H}_{2} \mathrm{O}, \mathrm{O}_{2}\right)<1 \mathrm{ppm}\right)$.

Кристаллическая структура бинарного фосфида FeP содержит ядра ${ }^{31} \mathrm{P}(I=1 / 2, \quad \gamma / 2 \pi=17.235 \mathrm{MHz} / \mathrm{T})$, очень удобные для ЯМР исследований благодаря высокой ЯМР-интенсивности, отсутствию квадрупольного момента и $100 \%$ естественному содержанию. Это предоставляет уникальную возможность изучения магнитной структуры Fе-подрешетки и наведенных сверхтонких взаимодействий $\mathrm{Fe}-{ }^{31} \mathrm{P}$, используя спектроскопию ЯМР ядер ${ }^{31} \mathrm{P}$.

Измерения ЯМР ${ }^{31} \mathrm{P}$ проводились в магнитоупорядоченном состоянии при $1.55 \mathrm{~K}$ с использованием обычного фазового когерентного импульсного ЯМРспектрометра. Спектры ЯМР измерялись путем развертки магнитного поля на нескольких фиксированных частотах в широком диапазоне $11-140 \mathrm{MHz}$, сигнал был получен путем интегрирования огибающей спинового эха во временной области и усреднения по количеству сканирований, которое зависит от частоты. Для сравнения на частоте $80 \mathrm{MHz}$ спектр ЯМР ${ }^{31} \mathrm{P}$ измерялся также в парамагнитном состоянии при $155 \mathrm{~K}$. Чтобы избежать эффектов скин-слоя и уменьшения коэффициента качества резонансной цепи из-за высокой металлической проводимости, порошковый образец фиксировался в парафине. Это также предотвращает переориентацию зерен образца в приложенном поле. Кроме того, ЯМР ${ }^{31} \mathrm{P}$ в нулевом поле измерялся при $4.2 \mathrm{~K}$ с использованием техники спин-эхо с пошаговым изменением частоты.

\section{3. Спектр ЯMP ${ }^{31} \mathrm{P}$ в FeP в нулевом поле}

ЯМР-спектр ядер ${ }^{31} \mathrm{P}$ в FeP, измеренный при нулевом внешнем магнитном поле при $4.2 \mathrm{~K}$, представлен на 


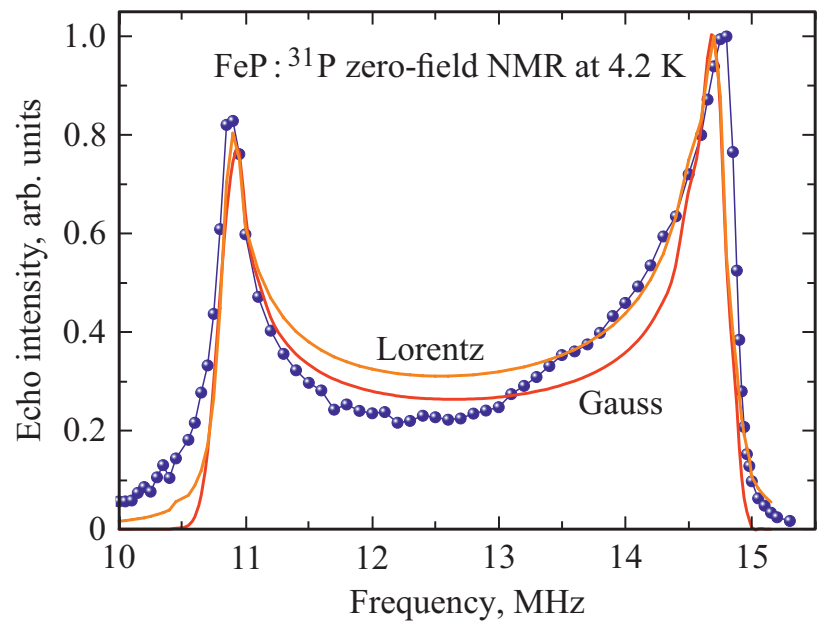

Рис. 1. Спектр ЯМР ядер ${ }^{31} \mathrm{P}$ в FеР в нулевом поле при $4.2 \mathrm{~K}$ : кружками обозначены экспериментальные данные, кривые результаты модельной обработки спектров в рамках модели геликоида (1) с параметром ангармоничности $m=0.32$ и с использованием гауссовой или лоренцевой форм индивидуальных линий.

рис. 1. Он демонстрирует очень широкое распределение интенсивности в диапазоне приблизительно от 10 до $15.3 \mathrm{MHz}$ с асимметричной двух-рожковой (doublehorn) формой с краевыми пиками, расположенными на 10.9 и $14.8 \mathrm{MHz}$. Разделив значения частоты пиков на $\gamma\left({ }^{31} \mathrm{P}\right) / 2 \pi=17.235 \mathrm{MHz} / \mathrm{T}$, сразу получим „крайние“ значения локального поля в позициях фосфора: $H_{\text {loc }}\left({ }^{31} \mathrm{P}\right)=0.63$ и $H_{\text {loc }}\left({ }^{31} \mathrm{P}\right)=0.86$ Т для левого и правого пика соответственно. Кроме того, в отличие от обычных коллинеарных антиферромагнетиков, где обычно наблюдаются изолированные линии ЯМР в нулевом поле на немагнитных атомах, асимметричный профиль с двумя рожками ${ }^{31} \mathrm{P}$ в FeP (рис. 1) однозначно указывает на квазинепрерывное распределение локального магнитного поля на ядрах ${ }^{31} \mathrm{P}$, типичное для полей, наводимых анизотропными ангармоническими магнитными спиралями. Так, спектры эффекта Мессбауэра ${ }^{57} \mathrm{Fe}$ в FeP хорошо описывались в предположении существования ангармонической магнитной спирали в Fе-подрешетке, распространяющейся вдоль $c$-оси [6], для которой

$$
\cos \theta(z)=\left[ \pm \frac{4 K(m)}{\lambda} z, m\right]
$$

где $\theta(z)$ - угол ориентации локального магнитного момента спирали в $a b$-плоскости, зависящий от координаты $z$ вдоль $c$-оси, [...] - эллиптическая функция Якоби, $K(m)$ - эллиптический интеграл первого рода, $\lambda$ - период спирали, а $m$ - параметр ангармонизма [7].

На рис. 1 представлены расчетные спектры ЯМР ядер ${ }^{31} \mathrm{P}$ в нулевом поле, рассчитанные с использованием соотношения (1) по методике [7,8] с использованием гауссовой и лоренцевой формы индивидуальных линий. Наилучшее соответствие теоретического и эксперимен- тального спектров ЯМР получено для параметра ангармонизма $m=0.32$ и лоренцевой формы индивидуальных линий. Интересно, что экспериментальный спектр ЯМР ядер ${ }^{31} \mathrm{P}$ в нулевом поле одинаково хорошо описывается и в простейшей модели ангармонической спирали

$$
\theta(z)=q z+k_{2} \sin 2 q z,
$$

где $q-$ волновой вектор спирали, $k_{2}=0.05-$ параметр анизотропии (ангармонизма) спирали.

\section{4. Спектр ЯMP ${ }^{31} \mathrm{P}$ в FeP во внешнем поле}

Первые результаты измерения спектра ЯМР ${ }^{31} \mathrm{P}$ в $\mathrm{FeP}$ во внешнем поле приведены в работе [6]. Спектр, измеренный при фиксированной частоте $F=80 \mathrm{MHz}$ $\left(B_{L}=4.642 \mathrm{~T}\right)$ в парамагнитном состоянии при $155 \mathrm{~K}$, очень узкий $\left(\mathrm{FWHM} \sim 6 \cdot 10^{-2} \mathrm{kOe}\right.$, однако при уменьшении температуры ниже $T_{\mathrm{N}} \approx 120 \mathrm{~K}$ ширина линии ЯМР ${ }^{31} \mathrm{P}$ становится больше на два порядка $(\mathrm{FWHM} \sim 11.6(4) \mathrm{kOe})$ из-за появления сверхтонкого поля с величиной около $1 \mathrm{~T}$, наводимого атомами $\mathrm{Fe}$. Чтобы более детально изучить наблюдаемую полевую зависимость формы линии ЯМР ${ }^{31} \mathrm{P}$, мы выполнили серию измерений спектров на образце порошка $\mathrm{FeP}$ в магнитоупорядоченном состоянии при $1.55 \mathrm{~K}$ на нескольких различных фиксированных частотах в диапазоне от 11 до $140 \mathrm{MHz}$. На рис. 2 приведены экспериментальные спектры для частот $11 \mathrm{MHz}$ (нижняя панель) и $120 \mathrm{MHz}$ (верхняя панель).

На самой низкой частоте $11 \mathrm{MHz}\left(B_{L}=0.638 \mathrm{~T}\right.$, рис. 2, нижняя панель) наибольшая интенсивность эха наблюдалась уже при внешнем магнитном поле $B=0$. При увеличении внешнего поля интенсивность эха быстро уменьшается с двумя различными особенностями около

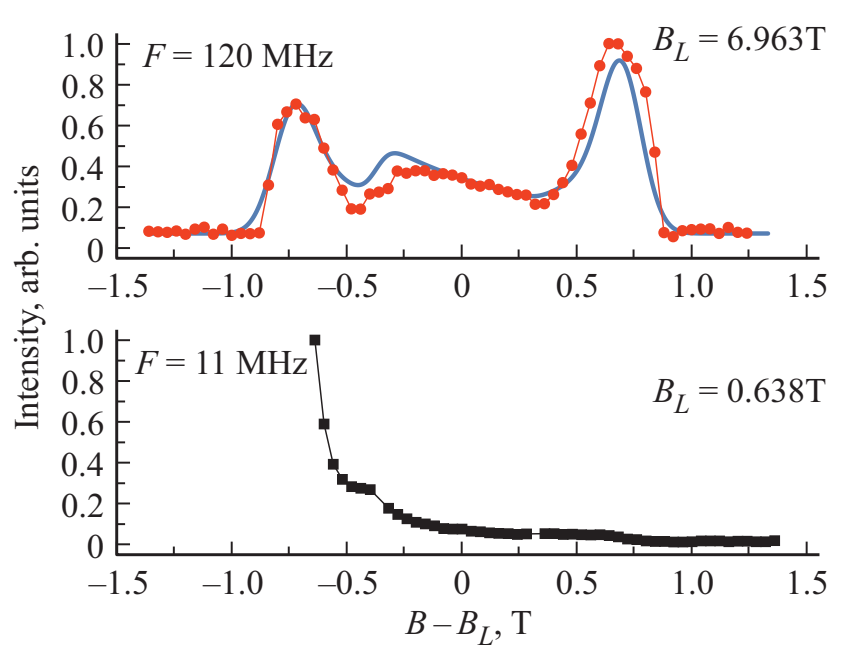

Рис. 2. Спектр ЯМР ядер ${ }^{31} \mathrm{P}$ в FеР во внешнем поле при $1.55 \mathrm{~K}$ при двух фиксированных частотах. Кривые - результат теоретической обработки спектров в модели суперпозиции. 
$B-B_{L}=-0.4 \mathrm{~T}$ и $B-B_{L}=0.6 \mathrm{~T}$. Спектр ЯМР ${ }^{31} \mathrm{P}$ на частоте $60 \mathrm{MHz}\left(B_{L}=3.48 \mathrm{~T}\right)$, измеренный ранее [6], имеет трапецеидальную форму, близкую к типичным для спектров ЯМР поликристаллических образцов [6]. Сигнал ЯМР в нулевом поле отсутствует, интенсивность эха распределена в широком интервале между симметричными пределами $B-B_{L}=-0.9 \mathrm{~T}$ и $B-B_{L}=+0.9 \mathrm{~T}$, тем самым указывая на величину 0.9 Т как на максимальное значение наведенного магнитного поля на ядрах

${ }^{31}$ Р. Форма сигнала ЯМР вполне удовлетворительно описывалась в рамках простой теории формы линии ЯМР в порошкообразном образце в предположении единой величины локального поля для всех ядер [6,9]

$$
F(H) \propto \int_{H_{<}}^{H_{>}} \frac{H^{2}-H_{\mathrm{loc}}^{2}+H_{\mathrm{res}}^{2}}{H_{\mathrm{loc}} H^{2}} \frac{1}{\delta} \exp \left[-\frac{(h-H)^{2}}{2 \delta^{2}}\right] d h,
$$

где $H_{>}=H_{\text {res }}+H_{\text {loc }}, H_{<}=H_{\text {res }}-H_{\text {loc }}, H_{\text {res }}=\omega_{0} / \gamma-$ резонансное поле, $H_{\mathrm{loc}}$ - локальное поле на ядре, $H$ - внешнее поле, $\delta$ - полуширина индивидуальной линии ЯМР.

Дальнейшее увеличение частоты и соответствующего внешнего магнитного поля приводит к резкой трансформации трапецеидального спектра ЯМP ${ }^{31} \mathrm{P}$ в FeP c появлением вместо правого и левого плеча прежней трапеции ярко выраженных пиков с широким максимумом между ними. Так, на частоте $120 \mathrm{MHz}\left(B_{L}=6.963 \mathrm{~T}\right)$ два боковых пика преобразуются в характерные асимметричные краевые пики, а широкая центральная часть имеет относительно небольшую амплитуду (см. рис. 2). Следует отметить два важных момента. Во-первых, в широком диапазоне измеренных частот спектральная интенсивность ЯМР ${ }^{31} \mathrm{P}$ распределена в одних и тех же симметричных пределах от $B-B_{L} \approx-0.7 \mathrm{~T}$ и $B-B_{L} \approx+0.7 \mathrm{~T}$, что указывает на сохранение максимальной абсолютной величины $\approx 0.7 \mathrm{~T}$ наведенного локального магнитного поля на ядрах ${ }^{31} \mathrm{P}$ независимо от трансформации магнитной структуры в магнитном поле. Во-вторых, асимметрия особенностей интенсивности меняется с увеличением поля: при низких полях более интенсивна левая особенность спектра, тогда как при высоких полях более интенсивным является правый пик. Наблюдаемая трансформация спектра ЯМР ${ }^{31} \mathrm{P}$ в FeP из трапецеидальной формы при низких внешних полях к асимметричной форме с двумя рогами при высоких полях скорее всего является свидетельством в пользу спин-переориентационного перехода типа спин-флоп в $\mathrm{FeP}$. Действительно, в случае индуцированного спинфлоп-перехода плоскости спирали во всех кристаллических зернах образца переориентируются в направлении внешнего поля и поведение порошкообразного образца в „магнитном отношении“ напоминает монокристалл. Хорошо известно, что „двухрожковая“ (double-horn) форма сигнала типична для ЯМР на ядрах немагнитных атомов в монокристаллах гелимагнетиков, таких как ${ }^{6,7} \mathrm{Li}$ в $\mathrm{LiCu}_{2} \mathrm{O}_{2}[10,11]$, или ${ }^{13} \mathrm{Na}$ в $\mathrm{NaCu}_{2} \mathrm{O}_{2}$ [12].
B простейшей модели спин-флоп-фазы в FeP предполагается, что магнитные моменты железа в спирали вращаются в плоскости, перпендикулярной внешнему полю Н. Однако в этом случае для объяснения симметричной „двухрожковой“ формы сигнала ЯМР необходимо предположить предельно анизотропное наведенное сверхтонкое взаимодействие $\mathrm{Fe}-{ }^{31} \mathrm{P}$, при котором локальное поле на ядрах ${ }^{31} \mathrm{P}$ ориентировано перпендикулярно плоскости спирали, то есть коллинеарно с внешним полем, а его величина зависит от ориентации магнитных моментов железа в спирали

$$
H_{\mathrm{loc}}=H_{\mathrm{int}} \cos \theta(z),
$$

где для угла $\theta(z)$ можно использовать выражение (2). Необычная форма линии ЯМР (рис. 2) с довольно четко выделенными пиками различной амплитуды и широким несимметричным максимумом между ними может быть объяснена в предположении, что основная доля Fе-спиралей находится в спин-флоп-фазе, но при этом имеется относительно небольшая доля спиралей с ЯМР-откликом, типичным скорее для поликристалла. Такая ситуация вполне возможна в условиях спин-флопперехода первого рода. Некоторая трудность возникает с объяснением различия амплитуд левого и правого пиков. Формально это можно объяснить включением в выражение (2) для угла $\theta(z)$ дополнительного ангармонизма

$$
\theta(z)=q z+k_{1} \sin q z+k_{2} \sin 2 q z .
$$

Форма линии ЯМР определяется плотностью распределения значений поля $H: g(z) \propto|d H(z) / d z|^{-1}$ и индивидуальной формой линии. После несложной алгебры получим

$$
\begin{aligned}
F(H) \propto & \int_{H_{<}}^{H_{>}} \frac{H_{\mathrm{int}}^{2}\left[H_{\mathrm{int}}^{2}-\left(H_{\mathrm{res}}-H\right)^{2}\right]^{-1 / 2}}{\left[H_{\mathrm{int}}^{2}+k_{1}\left(H_{\mathrm{res}}-H\right)+2 k_{2}\left(2\left(H_{\mathrm{res}}-H\right)^{2}-H_{\mathrm{int}}^{2}\right)\right]} \\
& \times \frac{1}{\delta} \exp \left[-\frac{(h-H)^{2}}{2 \delta^{2}}\right] d h
\end{aligned}
$$

где $H_{>}=H_{\text {res }}+H_{\text {int }}, H_{<}=H_{\text {res }}-H_{\text {int }}$. На рис. 2 экспериментальный спектр ЯМР ${ }^{31} \mathrm{P}$ на частоте $120 \mathrm{MHz}$ описан суперпозицией основного вклада спин-флоп-фазы при $H_{\text {int }}=0.7 \mathrm{~T}, k_{1}=0.05, k_{2}=-0.40$ и относительно небольшого вклада с „поликристаллическим“ откликом (3) при $H_{\mathrm{int}}=0.35 \mathrm{~T}$.

\section{5. Заключение}

С целью исследования трансформации магнитной спиральной структуры в металлическом фосфиде железа FeP во внешнем поле мы провели исследование ЯМР ядер ${ }^{31} \mathrm{P}$ как в нулевом поле, так и путем развертки внешнего магнитного поля на нескольких фиксированных частотах. Форма ЯМР-линий постепенно изменяется с увеличением поля от трапециеидальной формы в низких полях до ярко выраженной асимметричной 
формы с двумя „рогами“ в сильных полях, характерноя для монокристаллических гелимагнетиков. Для описания формы спектров нами предложена простая модель, предполагающая „суперпозицию“ „порошкообразного“ вклада и вклада спин-флоп-фазы, удельный вес которых зависит от величины внешнего поля. Хорошее согласие модельных спектров ЯМР ${ }^{31} \mathrm{P}$ в FеР в нулевом и отличном от нуля внешнем поле с экспериментом указывает на то, что рассматриваемое нами достаточно грубое приближение может дать адекватное описание основных особенностей магнитной структуры и наведенных сверхтонких взаимодействий в $\mathrm{FeP}$.

\section{Список литературы}

[1] В.И. Митюк, Г.А. Говор, М. Будзински. Неорган. Материалы 49, 17 (2013).

[2] H.T. Cho, I.J. Park, I.B. Shim, C.S. Kim, S.J. Kim. J. Korean. Phys. Soc. 60, 1049 (2012).

[3] G.P. Felcher, F.A. Smith, D. Bellavance, A. Wold. Phys. Rev. B 3, 3046 (1971).

[4] L. Häggström, A. Narayanasamy. J. Magn. Magn. Mater. 30, 249 (1982).

[5] R.E. Bailey, J.F. Duncan. Inorg. Chem. 6, 1444 (1967).

[6] A.V. Sobolev, I.A. Presniakov, A.A. Gippius, I.V. Chernyavskii, M. Schädler, N. Büttgen, S.A. Ibragimov, I.V. Morozov, A.V. Shevelkov. J. Alloys Comp. 675, 277 (2016).

[7] А.В. Залесский, А.К. Звездин, А.А. Фролов, А.А. Буш. Письма в ЖЭТФ 71, 682 (2000).

[8] A.A. Gippius, A.V. Tkachev, N.E. Gervits, V.S. Pokatilov, A.O. Konovalova, A.S. Sigov. Solid State Commun. 152, 552 (2012).

[9] J. Kikuchi, K. Ishiguchi, K. Motoya, M. Itoh, K. Inari, N. Eguchi, J. Akimitsu. J. Phys. Soc. Jpn. 69, 2660 (2000).

[10] A.A. Gippius, E.N. Morozova, A.S. Moskvin, A.V. Zalessky, A.A. Bush, M. Baenitz, H. Rosner, S.-L. Drechsler. Phys. Rev. B 70, 1-4(R) (2004).

[11] A.A. Bush, N. Büttgen, A.A. Gippius, M. Horvatić, M. Jeong, W. Kraetschmer, V.I. Marchenko, Yu.A. Sakhratov, L.E. Svistov. Phys. Rev. B 97, 054428 (2018).

[12] A.A. Gippius, A.S. Moskvin, S.-L. Drechsler. Phys. Rev. B 77, 180403(R) (2008).

Редактор Ю.Э. Китаев 